\title{
AVALIAÇÃO DA AÇÃO ANTIOXIDANTE DE PRODUTOS NATURAIS NO BIODIESEL B100 (GLYCINE MAX).
}

\section{RESUMO}

\author{
Ricardo Sonsim de Oliveira ${ }^{1}$ \\ Edson Antônio da Silva ${ }^{2}$ \\ Paulo Rogério Pinto Rodrigues ${ }^{3}$ \\ Samuel Nelson Melegari Souza
}

Este trabalho teve como objetivo principal avaliar a ação antioxidante de alguns extratos etanólicos e produtos naturais no biodiesel B100 obtido a partir do óleo da soja (Glycine max). Foram avaliados os extratos etanólicos de Hortelã, Alecrim, Erva Mate, óleo de Candeia e o Saburá e para efeito de comparação os antioxidantes sintéticos (BTH) butilhidroxi-tolueno e (TBHQ) terc-butil-hidroquinona. Os ensaios de estabilidade oxidativa em teste acelerado foram realizados no Rancimat na temperatura de $110^{\circ} \mathrm{C}$. Foram realizados testes com biodiesel puro e com aditivos em duas concentrações: 1000ppm $(0,1 \%)$ e $5000 \mathrm{ppm}(0,5 \%)$. O biodiesel puro apresentou um tempo de indução de $2,56 \mathrm{~h}$, os melhores resultados com o biodiesel aditivado foram obtidos na concentração de $0,5 \%$ (5000ppm) para os extratos de Hortelã, TBHQ (sintético) e o Saburá, respectivamente, com os seguintes tempos de indução: 3,90, 6,73 e 13,87h. Foram realizados ensaios de oxidação do biodiesel com o Rancimat para avaliar o efeito da concentração dos antioxidantes do Saburá (natural) e o antioxidante sintético TBHQ em cinco concentrações diferentes $(0,10 \%, 0,25 \%, 0,50 \%, 1,00 \%$ e $2,00 \%)$. Os maiores tempos de oxidação foram obtidos com o biodiesel aditivado com o Saburá proveniente do pólen coletado pelas abelhas Jataí (Tetragonista angustula) na concentração de 0,5\% (5000ppm), com tempo de indução de 13,87h e a do TBHQ de 6,73h. Os resultados obtidos mostraram que o Saburá tem elevada capacidade antioxidante e pode ser utilizado como aditivo ao biodiesel.

Palavras-chave: Aditivos, estabilidade oxidativa, biodiesel, inibidores.

\begin{abstract}
The aim of the present work was to evaluate the antioxidant activity of some ethanol extract sand natural products on B100 biodiesel obtained from soy bean oil (Glycine max). Ethanol extracts of Peppermint, Rosemary,Yerba Mate, oil and Candeia Sabura, as well as the synthetic antioxidants (BTH) butyl hydroxytoluene (TBHQ) tert-butyl hydroquinone, were evaluated for further comparison. Experiments of oxidative stability inaccelerated test were carried out at a temperature of $110^{\circ} \mathrm{C}$ by the Rancimat method. Tests were conducted with pure biodiesel and additives at two concentrations: $1000 \mathrm{ppm}(0.1 \%)$ and $5000 \mathrm{ppm}(0.5 \%)$ and pure biodiesel showed an induction time of $2.56 \mathrm{~h}$. The best results were obtained with biodiesel additive at $0.5 \%(5000 \mathrm{ppm})$ for Mint extracts, TBHQ (synthetic) and Sabura, respectively, with the following induction times: 3.90, 6.73 and13.87h. Oxidation tests were performed with the Rancimat biodiesel in order to evaluate the effect of antioxidant concentration of Sabura (natural) and the synthetic antioxidant TBHQ at five different concentrations $(0.10 \%, 0.25 \%, 0.50 \%, 1.00 \%$ and $2.00 \%)$. The higher oxidation times were obtained with the additive biodiesel Sabura from the pollen collected by bees Jatahy(Tetragonista angustula) at a concentration of $0.5 \%$ (5000ppm) with induction time of $13.87 \mathrm{~h}$ and TBHQ $6.73 \mathrm{~h}$. The results showed that the Sabura has high antioxidant capacity and can be used as an additive to biodiesel.
\end{abstract}

Keywords: Additives, oxidative stability, biodiesel, inhibitors.

\footnotetext{
${ }^{1}$ Fundação Assis Gurgacz - FAG. Email: ricardoquimica@ fag.edu.br

${ }^{2}$ Universidade Estadual do Oeste do Paraná -UNIOESTE. Programa de BIOENERGIA. edsondeq@ hotmail.com ${ }^{3}$ Universidade Estadual do Centro Oeste do Paraná - UNICENTRO. Programa de BIOENERGIA. prprodrigues@gmail.com

${ }^{4}$ Universidade Estadual do Oeste do Paraná - UNIOESTE Programa de BIOENERGIA. samuel.souza@ unioeste.br

ENGEVISTA, V. 16, n. 3, p.410-419, Setembro 2014
} 


\section{INTRODUÇÃO}

A alta demanda energética e os problemas ambientais produzem a necessidade de fontes de energias renováveis, sem limite de duração, e que podem trazer menor impacto ao meio ambiente. $\mathrm{O}$ biodiesel aparece como uma das alternativas para substituir o óleo diesel minimizando as dependências de importações de petróleo e reduzindo a poluição através da diminuição das emissões de gases poluentes (Geris et al., 2007).Alguns processos são responsáveis pela oxidação dos ésteres de ácidos graxos insaturados presentes no biodiesel. A estabilidade oxidativa, não depende apenas da composição química do biodiesel, depende também de quais condições foi submetido $o$ produto durante $o$ processamento, e do meio e maneira de estocagem. $\mathrm{O}$ grau de oxidação do biodiesel durante o período de armazenamento irá influenciar no potencial do combustível e esse não terá homogeneidade na queima, isso trará um atraso de ignição para os motores de ciclo diesel (Santos et al., 2010).Independente do tipo da rota tecnológica, a aceitação do biodiesel no mercado precisa ser assegurada e, para isso, é indispensável que esse produto esteja dentro das especificações aceitas para o seu uso. No Brasil os parâmetros de qualidade do biodiesel encontram-se normatizados pela portaria n²55 da ANP - Agência Nacional do Petróleo Gás Natural e Biocombústivel, sendo um dos parâmetros avaliados a estabilidade oxidativa; além disso, as características físico-químicas, do biodiesel a ser produzido e comercializado, precisam respeitar os limites da especificação proposta pela resolução $\mathrm{n}^{\circ}$ 42/2004 da ANP. Na oxidação do biodiesel são gerados vários compostos como aldeídos, cetonas, ácidos, peróxidos, polímeros, que modificam as propriedades do combustível afetando assim o funcionamento do motor(Santos et al., 2010).A estabilidade oxidativa deve ser entendida como um parâmetro essencial no controle da propriedade do biodiesel (Dantas et al., 2011).Segundo Jain e Sharma (2010) vários métodos têm sido reportados na literatura para avaliar a resistência à oxidação do biodiesel, dentre eles: o método petroOXY, PDSC e o
Rancimat, No método petroOXY a amostra é aquecida e submetida ao contato com oxigênio numa câmara, monitora-se o tempo necessário para que a amostra absorva $10 \%$ da pressão de oxigênio (Candeia, 2008). O P-DSC similar a técnica de calorimetria diferencial de varredura (DSC), porém sob alta pressão, essa técnica, pode ser determinada como uma técnica que mede as temperaturas e fluxo de calor associados aos eventos de transições dos materiais em função da temperatura e do tempo(Mothé; Azevedo, 2002). No Rancimat a estabilidade a oxidação do biodiesel é avaliada por meio do acompanhamento da condutividade elétrica das amostras que são expostas a presença de oxigênio em temperatura controlada (Lôbo et al., 2009).Para evitar ou retardar a degradação do biodiesel, dos óleos e gorduras, são empregados substâncias químicas denominados de antioxidantes, estes compostos agem impedindo a etapa inicial da auto oxidação e a formação de radicais livres, removendo-os do meio(Rios, 2004).Dentre os antioxidantes naturais mais empregados, podem ser citados os tocoferóis, os ácidos fenólicos e os extratos de plantas como alecrim e sálvia (Ramalho; Jorge, 2006). O tocoferol, por ser um dos melhores antioxidantes naturais, é largamente aplicado para inibir a oxidação dos óleos e gorduras comestíveis, prevenindo a oxidação dos ácidos graxos insaturados. Eles estão presentes de forma natural na maioria dos óleos vegetais e atualmente são também fabricados por síntese (Ramalho; Jorge, 2006). Os principais e mais conhecidos antioxidantes sintéticos são os polifenóis,o butil-hidroxianisol (BHA), o 2,6-di-terc-butilhidroxitolueno (BHT), o terc-butilhidroquino na (TBHQ) e o propil galato (PG),amplamente utilizados na indústria de alimentos e no biodiesel (Talcott et al., 2005). Desta forma, o presente trabalho tem como objetivo geral avaliar compostos naturais com potencial antioxidante para retardar a oxidação do biodiesel B100, obtido a partir do óleo da soja (Glycine max) e comparar sua atividade em relação aos antioxidantes sintéticos (TBHQ e BHT). 


\section{METODOLOGIA}

\subsection{PREPARO DO BIODIESEL}

O biodiesel metílico de soja foi sintetizado usando como catalisador hidróxido de potássio. Foram utilizados nos experimentos óleo de soja comercial da marca COAMO, álcool anidro (Tedia do
Brasil) e hidróxido de potássio (Vetec). A reação foi conduzida na temperatura de $50^{\circ} \mathrm{C}$, com $3 \%$ de razão mássica (catalisador/óleo), e razão volumétrica (óleo/álcool) de $30 \%$ v/v e tempo reacional de 60 minutos(Figura 1).

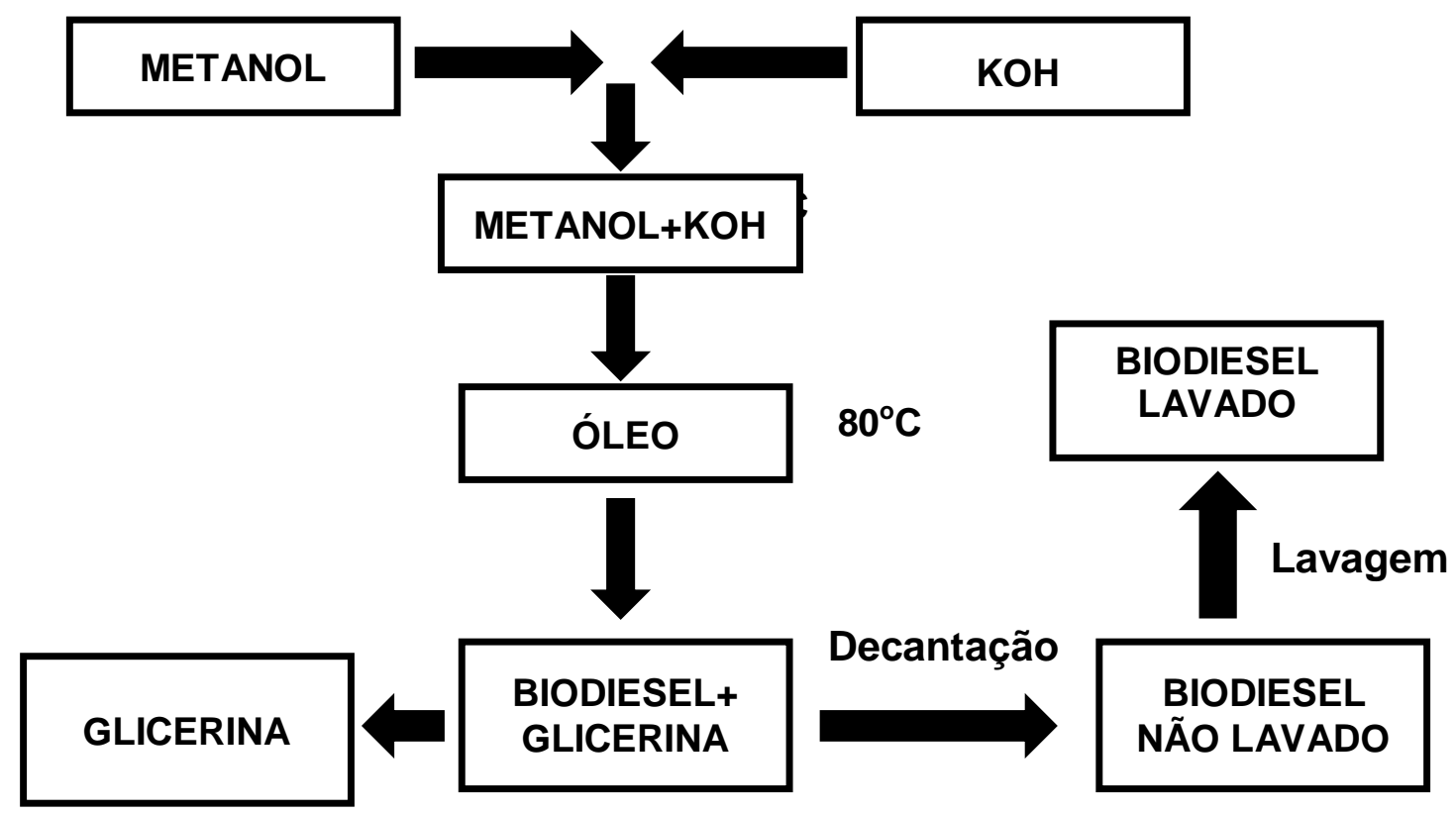

Figura 1 - Fluxograma da produção de biodiesel.

Fonte: Adaptado de Gallina et al., 2010.

Após o término da reação, transferiu-se a solução para um funil de decantação por um tempo aproximado de $24 \mathrm{~h}$. Ocorrido a decantação, realizou-se $\mathrm{o}$ processo de separação do óleo transesterificado Biodiesel (fase superior) e do glicerol ou glicerina (fase inferior). $\mathrm{O}$ biodiesel (fase superior) foi lavado com uma solução de concentração $0,01 \mathrm{~mol}$. $\mathrm{L}^{-1}$ de ácido acético por três vezes e separado por decantação. Assim, a fase aquosa (inferior) é eliminada e a fase orgânica (superior) é armazenada. Esta fase representa o biodiesel lavado e neutralizado. Para caracterização do biodiesel foram realizados os seguintes testes: massa específica, pelo método que é nomeado como ASTM - D1298 e NBR 7148 (ANP, 2011); cor visual e aspecto, feitas em um recipiente transparente contra a luz observando cuidadosamente a presença de impurezas, e/ou água no fundo do recipiente e a coloração do produto (ANP, 2011); e o potencial hidrogeniônico $(\mathrm{pH})$, atendendo as especificações exigidas pela ANP prevista na norma ASTM D 1125.

\subsection{PREPARO DOS EXTRATOS NATURAIS}

Antes das extrações, as folhas de erva mate, alecrim e hortelã foram secas em estufa digital para secagem Inox, 30L(modelo SP400) a temperatura de $50^{\circ} \mathrm{C}$. Após o processo de secagem, as folhas foram trituradas a fim de aumentar sua área de contato com o solvente, para isto, empregou-se um cadinho e um bastão de porcelana efetuando a moagem manualmente. Em seguida, adicionou-se álcool etílico anidro (99,9\% Labsynth) em uma proporção de 1:20 massa/volume. A solução alcoólica foi deixada em repouso por um período de $24 \mathrm{~h}$, depois filtrada e armazenada, sob refrigeração, em frascos âmbar. O saburá, pólen fermentado e estocado pelas abelhas, foi coletado na região Noroeste do Paraná, no distrito Porto Camargo do município de Icaraíma no final da tarde. Este coletado, foi produzido pelas 
abelhas da espécie Tetragonista angustula (Jataí sem ferrão).O material foi separado e transferido in natura para um recipiente de vidro com tampa de pressão e armazenado na geladeira, onde permaneceu à uma temperatura de 2 a $4^{\circ} \mathrm{C}$ conservando assim suas características.

O óleo bruto de candeia foi adquirido comercialmente para ser testado.

\subsection{ANTIOXIDANTES SINTÉTICOS}

Foram utilizados os antioxidantes sintéticos com a finalidade da comprovação da extensão da vida útil do biodiesel. Estes, foram adquiridos no mercado nacional, sendo o BHT e o TBHQ 99\% da marca Embranox fabricado por Danisco.

\subsection{PREPARO DO BIODIESEL PARA ENSAIOS DE OXIDAÇÃO NO RANCIMAT}

Cerca de $50 \mathrm{~g}$ do biodiesel foram transferidos para um béquer e colocado em um agitador magnético, modelo IKA RH Basic KT/C, com aquecedor a $50{ }^{0} \mathrm{C} . \mathrm{Em}$ seguida foi adicionado uma massa conhecida das substâncias ou extratos avaliados. Após 20 minutos de agitação o biodiesel aditivado foi retirado do agitador, deixado em repouso à temperatura ambiente e, posteriormente, armazenado em frascos âmbar.

Foram realizados ensaios preliminares com biodiesel puro e com biodiesel aditivado com os extratos etanólicos: erva mate, alecrim e hortelã, óleo de candeia (in natura), pólen e os antioxidantes sintéticos TBHQ e BHT. As concentrações utilizadas foram de 1000ppm e 5000pm.

Posteriormente foram realizados ensaios com um antioxidante natural e outro sintético, para avaliar o efeito da concentração $(0,1000,2500,5000,10000$ e 20000ppm) na estabilidade oxidativa do biodiesel. Os antioxidantes empregados foram selecionados a partir dos resultados nos testes preliminares que avaliaram diferentes compostos.

\subsection{ENSAIOS DE ESTABILIDADE OXIDATIVA NO RANCIMAT}

Nos testes de estabilidade oxidativa acelerada com o Rancimat, a oxidação é induzida pela passagem de ar pela amostra, mantida à temperatura constante de $110^{\circ} \mathrm{C}$. Os produtos voláteis oriundos da reação são coletados em água destilada e monitora-se a condutividade elétrica da fase líquida. A resposta obtida no Rancimat é uma curva de condutividade elétrica versus tempo, que permite determinar o tempo de indução oxidativa a partir da interseção das retas tangentes à curva. Estes resultados são fornecidos automaticamente pelo Software do equipamento. A estabilidade à oxidação foi medida, segundo a norma EN 14112, utilizando amostras de $3 \mathrm{~g}$ de biodiesel as quais foram analisadas sob aquecimento com temperatura de $110^{\circ} \mathrm{C}$ e fluxo de ar constante de $10 \mathrm{Lh}^{-1}$. Os ensaios referentes à estabilidade da oxidação realizados no Rancimat foram feitos em duplicatas nos testes preliminares e em triplicatas para avaliar o efeito da concentração.

\section{RESULTADOS E DISCUSSÕES}

O biodiesel metílico foi preparado a partir do óleo da soja empregando catálise homogênea com hidróxido de potássio. $\mathrm{O}$ biodiesel antes da lavagem apresentava um potencial hidrogeniônico $(\mathrm{pH})$ próximo de 8 , porém para a realização das aditivações e posteriores ensaios, o biodiesel foi lavado com uma solução de ácido acético de concentração $0,01 \mathrm{~mol} \cdot \mathrm{L}^{-1}$. Após a lavagem o $\mathrm{pH}$ do biodiesel foi neutralizado $(\mathrm{pH}=7)$, atendendo dessa forma as especificações exigidas pela ANP, cujo valor previsto é $\mathrm{pH}=7 \pm 1$. A lavagem do biodiesel permite a separação da fase aquosa e a fase orgânica (biodiesel). A condutividade nessa fase (orgânica) se torna nula, o máximo permitido são $350 \mu \mathrm{S} \cdot \mathrm{m}^{-1}$. Nos testes preliminares de estabilidade oxidativa foram testados a ação antioxidante de sete aditivos, sendo dois sintéticos e cinco naturais, em dois níveis de concentração $0,1 \%$ (1000ppm) e $0,5 \%$ (5000ppm) todos em duplicata. Os resultados obtidos nos ensaios de oxidação acelerada do biodiesel no Rancimat são apresentadas na Tabela 1.Observa-se que o biodiesel puro e os extratos etanólicos não atenderam as especificações da ANP, devido ao baixo potencial da capacidade antioxidante, isso também foi verificado com o BHT (antioxidante sintético), uma vez que o 
valor do tempo de indução a $110^{\circ} \mathrm{C}$ para o biodiesel puro e aditivado deveria ter um valor mínimo de $6 \mathrm{~h}$ para o tempo de indução (EN 14112, 2003).

Resultados semelhantes da ação antioxidante do BHT foram obtidos por Ferrari e Souza (2009) que testaram sua ação no biodiesel de girassol numa concentração de $5 \%$ (5000ppm) e verificaram um período de indução de 3,00h.Testaram também o TBHQ e o tempo foi de $6,50 \mathrm{~h}$ atendendo a norma $\mathrm{EN}$ 14112 , 2003. O valor do período de indução da amostra de concentração $0,5 \%$ apresentou um valor semelhante ao mesmo composto (TBHQ) aplicado no biodiesel de girassol por Ferrari e Souza (2009), como citado, esse período foi de $6,5 \mathrm{~h}$ no biodiesel de girassol e de $6,73 \mathrm{~h} \pm 0,05$ no biodiesel da soja utilizado no presente trabalho.

A literatura mostra diversos estudos relacionados ao uso do TBHQ em biodiesel de soja. Estes estudos indicam que o TBHQ possui a maior capacidade em retardar o processo oxidativo em relação aos demais antioxidantes sintéticos BHT, BHA ou PG (Damasceno, 2011).

Araujo, Moura e Chaves (2010), submeteram o biodiesel metílico de garampara ao tratamento com dois antioxidantes sintéticos, TBHQ e BHT, e observaram um aumento do período de indução com o aumento da concentração do antioxidante, sendo que o TBHQ apresentou melhores resultados do que o BHT, nas mesmas concentrações, em concordância com o relatado na literatura.

Este resultado mostrou-se semelhante ao desta pesquisa e, diante de todas as amostras aditivadas e testadas na primeira etapa, apenas uma atendeu a especificação da ANP como antioxidante natural, o Saburá (pólen apícola coletado pelas abelhas Jataí Tetragonista angustula). A Figura 2 mostra a média do período de indução em função do tipo de amostra a $0,1 \%$ ou $1000 \mathrm{ppm}$.

Tabela 1 - Estabilidade oxidativa no Rancimat a $100^{\circ} \mathrm{C}$.

\begin{tabular}{|l|r|r|c|}
\hline $\begin{array}{l}\text { Amostras de } \\
\text { Biodiesel com seus } \\
\text { respectivos Aditivos }\end{array}$ & $\begin{array}{c}\text { Concentração do } \\
\text { Aditivo (\%) m/m }\end{array}$ & $\begin{array}{c}\text { Concentração do } \\
\text { Aditivo (ppm) }\end{array}$ & $\begin{array}{c}\text { Período de } \\
\text { Indução(h) }\end{array}$ \\
\cline { 2 - 4 } & Porcentagem & Partes Por Milhão & $\mathbf{1 1 0} \mathbf{~} \mathbf{C}^{\mathbf{C}}$ \\
\hline Controle Puro & - & - & $2,56 \pm 0,06$ \\
\hline Candeia & 0,1 & 1000 & $1,64 \pm 0,08$ \\
\hline Candeia & 0,5 & 5000 & $1,71 \pm 0,07$ \\
\hline Extrato de Hortelã & 0,1 & 1000 & $2,71 \pm 0,09$ \\
\hline Extrato de Hortelã & 0,5 & 5000 & $3,90 \pm 0,06$ \\
\hline Extrato de Alecrim & 0,1 & 1000 & $1,94 \pm 0,09$ \\
\hline Extrato de Alecrim & 0,5 & 5000 & $2,25 \pm 0,05$ \\
\hline Extrato de Erva Mate & 0,1 & 1000 & $1,38 \pm 0,09$ \\
\hline Extrato de Erva Mate & 0,5 & 5000 & $2,16 \pm 0,06$ \\
\hline Saburá & 0,1 & 1000 & $11,28 \pm 0,09$ \\
\hline Saburá & 0,5 & 5000 & $13,87 \pm 0,06$ \\
\hline BHT & 0,1 & 1000 & $3,10 \pm 0,08$ \\
\hline BHT & 0,5 & 5000 & $4,32 \pm 0,09$ \\
\hline TBHT & 0,1 & 1000 & $3,96 \pm 0,07$ \\
\hline TBHT & 0,5 & 5000 & $6,32 \pm 0,09$ \\
\hline
\end{tabular}




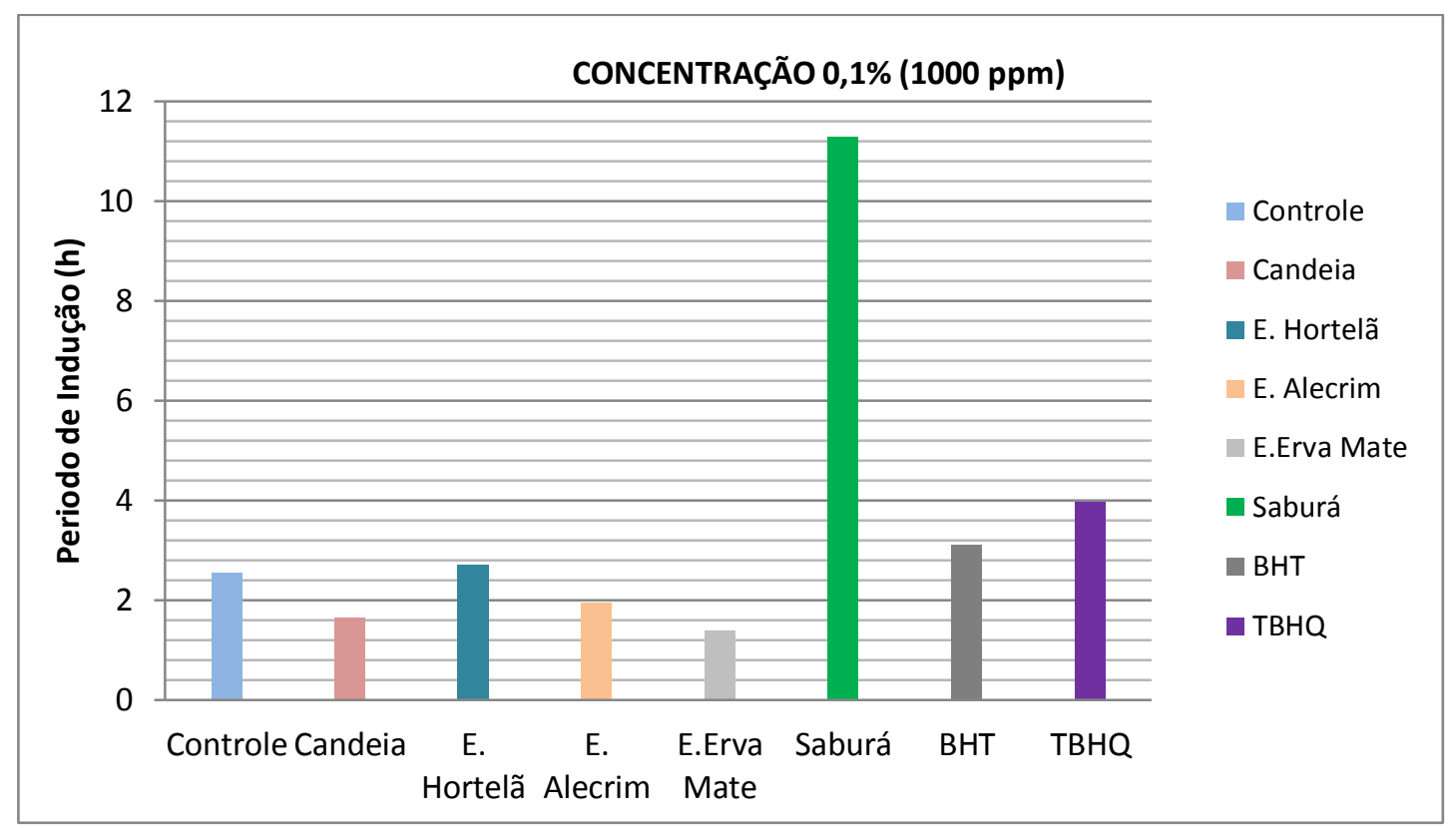

Figura 2 - Período de indução do Biodiesel puro e aditivado 1000ppm.

Quando as amostras foram aditivadas e preparadas em uma concentração de $0,1 \%$ ou 1000ppm com diferentes tipos de antioxidantes, o maior período de indução foi de $11,28 \pm 0,09$, obtido com a mistura do pólen apícola (Saburá) ao biodiesel B100 e o menor período de indução foi de $1,38 \pm 0,09$ do extrato de erva mate que acelerou a oxidação, ou seja, favoreceu a oxidação do biodiesel. A amostra controle que é o biodiesel puro teve um período de oxidação maior que a mistura com candeia, alecrim e erva mate, já a hortelã teve uma pequena ação antioxidante ,incompatível com os parâmetros determinados pela ANP.

A Figura 3 mostra a média do período de indução em função do tipo de amostra, na concentração de $0,5 \%$ ou 5000 ppm.

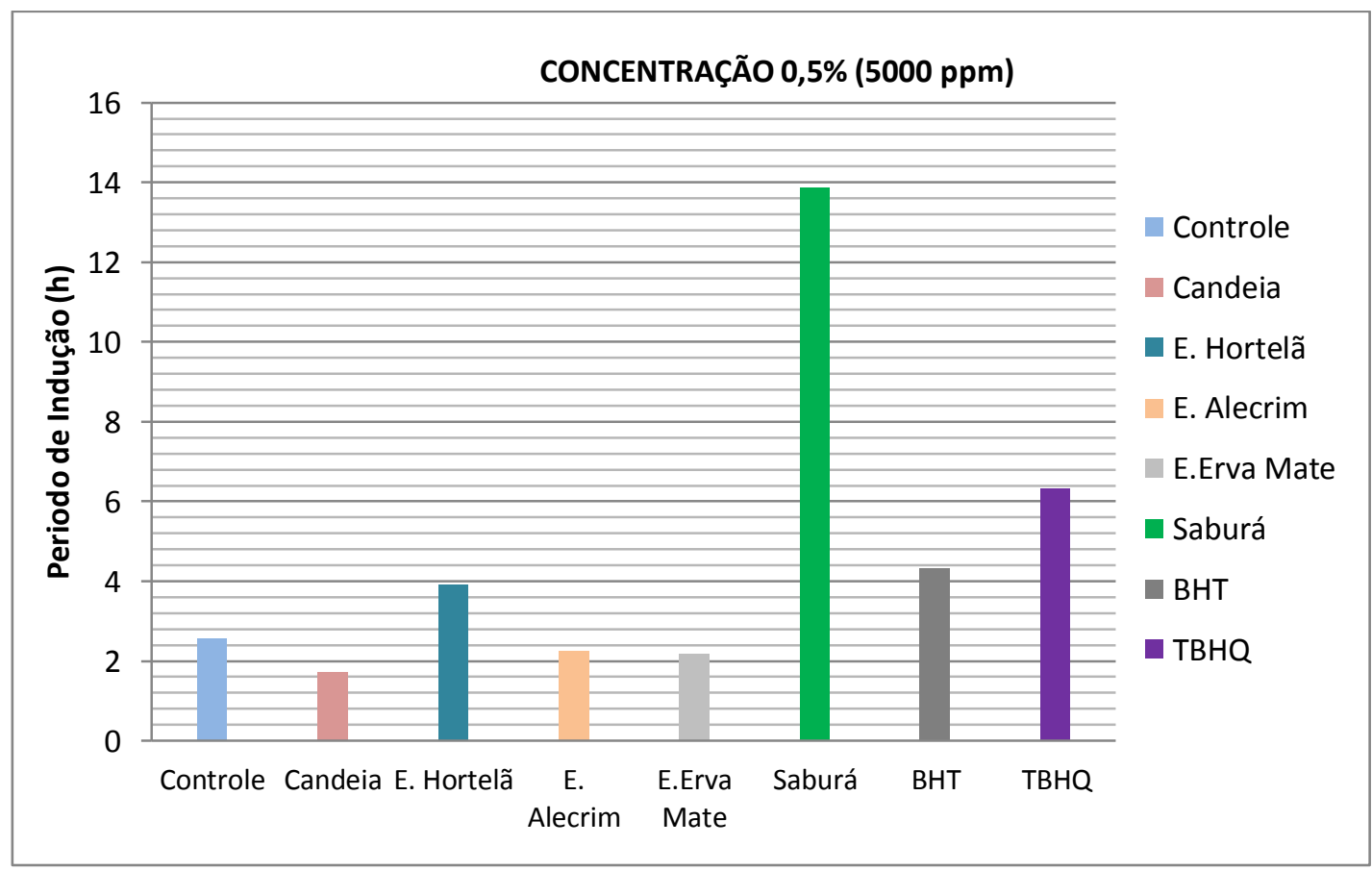

Figura 3 - Período de indução do Biodiesel puro e aditivado 5000ppm. 
Quando as amostras foram aditivadas e preparadas com uma concentração de $0,5 \%$ ou 5000ppm, usando diferentes tipos de antioxidantes, o maior período de indução foi de $13,87 \mathrm{~h} \pm 0,06$, obtido com a mistura do pólen apícola (Saburá) ao biodiesel B100 e, o menor período de indução foi de $1,71 \pm 0,07$, com a mistura do óleo de candeia ao biodiesel B100.

Os ensaios realizados em diferentes concentrações, conforme mostrado na Tabela 1, sugerem que todas as amostras melhoraram seu potencial antioxidante com o aumento da concentração. A hortelã também se apresentou como antioxidante porém com um período de indução inferior às6 horas determinadas pelas normas da ANP.

Numa segunda etapa foi avaliado o efeito da concentração do Saburá e do TBHQ na ação antioxidante do biodiesel de soja.

Os valores utilizados na produção das amostras com o TBHQ, suas concentrações e o período de indução verificado no Rancimat a $110^{\circ} \mathrm{C}$ seguindo a norma EN 14112 de 2003são apresentados na Tabela 2.

Nota-se queo período de indução maior foi obtido na amostra 4 que apresenta um nível de concentração de 5000ppm. Os ensaios das amostras com concentrações inferiores à 5000ppm também tiveram ação antioxidante no biodiesel, com o período de indução maior, em relação ao controle, mas insuficiente para serem utilizadas como aditivos no biodiesel.
A utilização do TBHQ como antioxidante sintético comparativo teve como parâmetro outros trabalhos que revelam ser ele o melhor e mais eficiente antioxidante sintético comercial para óleos.

Ramalho e Jorge (2006) concluíram em seu trabalho que o antioxidante TBHQ para os óleos vegetais, foi o de maior potencial antioxidante, inclusive em altas temperaturas.

$\mathrm{Na}$ faixa de concentração avaliada, verificou-se que a concentração $0,5 \%$ ou 5000 ppm do TBHQ foi a que apresentou os melhores resultados para retardar a oxidação do biodiesel. Estudos de Obadiah et al. (2012) que avaliaram a estabilidade oxidativa do biodiesel com o TBHQ e outros antioxidantes sintéticos demonstraram uma relação linear entre o tempo de indução e a concentração dos aditivos, a faixa de estudo foi de 500 a 3000ppm.Resultados estes, semelhantes aos obtidos no presente trabalho para a faixa de concentração de 1000 a 5000ppm.Para níveis superiores a 5000ppm não foram encontrados relatos na literatura do efeito da concentração do TBHQ na estabilidade oxidativa do biodiesel.

$\mathrm{Na}$ Tabela 3 estão apresentados os valores utilizados na produção das amostras com o Saburá coletado na região Noroeste do Paraná, no distrito Porto Camargo do município de Icaraíma. Além das suas concentrações e dos períodos de indução verificados no Rancimat a $110^{\circ} \mathrm{C}$ seguindo a norma EN 14112 de 2003.

Tabela 2 - Tempo de indução do TBHQ em diferentes concentrações.

\begin{tabular}{|c|c|c|c|}
\hline $\begin{array}{c}\text { AMOSTRA } \\
(\mathbf{m} / \mathbf{m})\end{array}$ & $\begin{array}{c}\text { PORCENTAGEM } \\
(\boldsymbol{\%})\end{array}$ & $\begin{array}{c}\text { CONCNETRAÇÃO } \\
(\mathbf{p p m})\end{array}$ & $\begin{array}{c}\text { PERÍODO } \\
\text { DEINDUÇÃO } \\
(\mathbf{h})\end{array}$ \\
\hline 1 & 0,00 & 0000 & $2,53 \pm 0,04$ \\
\hline 2 & 0,10 & 1000 & $3,37 \pm 0,07$ \\
\hline 3 & 0,25 & 2500 & $4,02 \pm 0,08$ \\
\hline 4 & 0,50 & 5000 & $6,73 \pm 0,05$ \\
\hline 5 & 1,00 & 10000 & $0,91 \pm 0,09$ \\
\hline 6 & 2,00 & 20000 & $0,47 \pm 0,10$ \\
\hline
\end{tabular}


Tabela 3 - Tempo de indução do Saburá (Noroeste) em diferentes concentrações.

\begin{tabular}{|c|c|c|c|}
\hline $\begin{array}{c}\text { AMOSTRA } \\
(\mathbf{m} / \mathbf{m})\end{array}$ & $\begin{array}{c}\text { PORCENTAGEM } \\
(\boldsymbol{\%})\end{array}$ & $\begin{array}{c}\text { CONCENTRAÇÃO } \\
(\mathbf{p p m})\end{array}$ & $\begin{array}{c}\text { PERÍODO } \\
\text { DE } \\
\text { INDUÇÃ O }(\mathbf{h})\end{array}$ \\
\hline 1 & 0,00 & 0000 & $2,53 \pm 0,07$ \\
\hline 2 & 0,10 & 1000 & $3,82 \pm 0,06$ \\
\hline 3 & 0,25 & 2500 & $4,11 \pm 0,09$ \\
\hline 4 & 0,50 & 5000 & $13,64 \pm 0,06$ \\
\hline 5 & 1,00 & 10000 & $0,96 \pm 0,09$ \\
\hline 6 & 2,00 & 20000 & $0,53 \pm 0,10$ \\
\hline
\end{tabular}

Os resultados apresentados na Tabela 2 mostram que a concentração do TBHQ teve um efeito significativo no tempo de oxidação, enquanto somente a amostra 4, com nível de concentração de 5000ppm,se enquadrou nas normas da ANP. Observase também que houve um aumento do tempo de indução oxidativa do biodiesel com a concentração do TBHQ até a concentração de 5000ppm, nos níveis maiores de concentração houve um decréscimo do tempo de indução oxidativa, sendo inclusive inferior ao do biodiesel puro (2,53 horas).

O Saburá apresentou um valor superior aos dois antioxidantes sintéticos. Observa-se que o resultado do período de indução obtido pelo Saburá na concentração de $0,5 \%$ foi superior a do antioxidante sintético TBHQ. Assim, esse composto pode ser considerado um antioxidante do biodiesel, pela norma da ANP e pela EN 14112 de 2003. Os resultados apresentados na Tabela 3 mostram que a concentração também altera o período de indução das amostras aditivadas como Saburá e que em concentrações superiores a $5000 \mathrm{ppm}$ ocorre uma aceleração da oxidação do biodiesel, com tempos de indução inferiores ao do biodiesel puro. Isso pode ser atribuído a um efeito misto,no qual a partir de um determinado nível de concentração o Saburá passa a atuar como catalisador da oxidação ou pró-oxidante com o aumento da concentração.

Considerando todas as amostras de biodiesel aditivadas e preparadas com diferentes níveis de concentrações do TBHQ e do Saburá, a que apresentou o maior período de indução foi a obtida com a mistura do pólen apícola (Saburá) ao biodiesel B100 na concentração de 0,5\% ou 5000ppm(13,64h士 0,06).O menor período de indução foi obtido na mistura, de maior concentração $2 \%$ ou $20000 \mathrm{ppm}$, do biodiesel ao TBHQ $(0,47 \mathrm{~h} \pm 0,10)$.

\section{CONCLUSÃO}

Neste trabalho foi avaliado o efeito antioxidante no biodiesel, preparado com uma mistura de diferentes extratos e compostos, por meio do teste de oxidação acelerada no Rancimat. Foi avaliado o biodiesel puro e aditivado com os extratos etanólicos: erva mate, alecrim e hortelã, óleo de candeia (in natura), pólen e os antioxidantes sintéticos TBHQ e BHT, em dois níveis de concentração 1000ppm e $5000 \mathrm{ppm}$. Os melhores resultados foram obtidos com o biodiesel aditivado com TBHQ e com saburá na concentração de 5000ppm, com tempo de indução de 6,73h e 13,64h, respectivamente. Também foi avaliado o efeito da concentração do TBHQ e do saburá na estabilidade oxidativa do biodiesel.

Verificou-se que ocorre um aumento do tempo de indução do biodiesel com o aumento da concentração destes compostos até o nível de 5000ppm de concentração. Acima deste, observou-se uma aceleração da oxidação do biodiesel, com tempo de indução inferior ao do biodiesel puro, demonstrando que nesta faixa de concentração estes compostos favorecem a reação de oxidação. Os resultados obtidos neste trabalho mostraram que o TBHQ e o saburá, nos níveis de concentração de 5000ppm, 
podem ser utilizados com aditivos no biodiesel.

\section{BIBLIOGRAFIA}

ANP - Agência Nacional do Petróleo Resolução ANP nº42, de 24/11/2004.

ANP - Agência Nacional do Petróleo Resolução ANP nº5, de 12/12/2011.

ARAÚJO, F. D. DA S.;MOURA,C. V. R. DE;CHAVES,M. H. Biodiesel metílico de Dipteryx lacunifera: preparação, caracterização e efeito de antioxidantes na estabilidade à oxidação. Química Nova, v. 33, n. 8, 1671-1676, 2010.

CANDEIA, R. A. Biodiesel de Soja: Síntese, Degradação e Misturas Binárias. Tese de Doutorado. Programa de PósGraduação em Química, UFPB.Paraíba, 2008.

DAMASCENO, S. S. Avaliação da Estabilidade Antioxidante dos Ácidos Caféico e Ferulíco no Controle da Estabilidade Oxidativa do Biodiesel de Soja. Dissertação de Mestrado.Programa de pós-graduação da UFPB. Paraíba, 2011.

DANTAS, M. B.; ALBUQUERQUE, A. R.; BARROS, A. K.; RODRIGUES FILHO, M. G.; ANTONIOSI FILHO, N. R.; SINFRÔNIO, F. S. M.; ROSENHAIM, R.; SOLEDADE, L. E. B.; SANTOS, I. M. G.; SOUZA, A.G. Evaluation of the oxidative stability of corn biodiesel. Fuel, v. 90, n. 1, 773-778, 2011.

EN 14112 - Fat and oil derivatives - Fatty acid methyl esters (FAME) Determination of oxidation stability (accelerated oxidation test), 2003.

FERRARI, R. A; SOUZA, W.L. Avaliação da estabilidade oxidativa de biodiesel de óleo de girassol com antioxidantes. Centro de Ciências e Qualidade de Alimentos, Instituto de Tecnologia de Alimentos. Campinas - SP, Brasil. Química Nova, v.32, n. 1, São Paulo, 2009.

ENGEVISTA, V. 16, n. 3, p.410-419, Setembro 2014
GALLINA, A. L.; STROPARO, E. C.; CUNHA, M. T.; RODRIGUES, P. R. P. A. A corrosão do aço inoxidável austenítico 304 em biodiesel. Revista Escola de Minas, v. 63, n. 1,71-75, Ouro Preto, 2010.

GERIS, R.; SANTOS, N. A. C. DOS; AMARAL, B. A.; MAIA, I. DE S.; CASTRO, V. D.; CARVALHO, J. R. M. Biodiesel de Soja - Reação de Trasesterificação para Aulas Práticas de Química Orgânica. Química Nova, v.30, n. 5, 1369-1373, 2007.

JAIN, S.; SHARMA, M. P. Stability of biodiesel and its blends: A review. Renewable and Sustainable Energy Reviews; v. 14, n. 2, 667-678, 2010.

LÔBO, I. P.; FERREIRA, S. L. C.; CRUZ, R. S. Biodiesel: parâmetros de qualidade e métodos analíticos. Química Nova, v. 32 n. 6, 1596-1608, 2009.

MOTHÉ, C. G.; AZEVEDO, A. D., Análise Térmica de Materiais. iEditora: São Paulo, 2002.

OBADIAH, A.; KANNAN, R.; RAMASUBBU, A.; KUMAR, S. V. Studies on the effect of antioxidants on the long-term storage and oxidation stability of Pongamia pinnata (L.) Pierre biodiesel. Fuel Processing Technology,v. 99, 56-63, 2012.

RAMALHO, V. C.; JORGE, N. Antioxidantes utilizados em Óleos, Gorduras e Alimentos Gordurosos. Química Nova, v. 29, n. 4, 755-760, 2006.

RIOS, M. A. F. Síntese de um Antioxidante Fosforado a partir do Cardanol Hidrogenado. Dissertação de Mestrado, Programa de Pós Graduação em Engenharia Química da Universidade Federal do Ceará, 2004. 
SANTOS, T. A.; RIBEIRO, C. L.; BRITO, B. O.; SOUZA, A. G.; CANDEIA, R. A.; SILVA, M. C. D. Biodiesel de Soja Aditivado com Cardanol - Efeito Antioxidante In: VII Congresso Brasileiro de Análise Térmica e Calorimetria, 25 a 28 de abril de 2010, São Pedro-SP, 2010.

TALCOTT, S. T.; DUNCAN, C. E.; POZO-INSFRAN, D. F.; GORBET, D.W. Polyphenolic and antioxidante changes during storange of normal, mid, and high oleic acid peanuts. Food Chemistry, v. 89, 77-84, 2005. 